Jahrbuch Dritte Welt: Daten, Úbersichten, Analysen - 1 (1983),

hrsg. vom Deutschen Úbersee-Institut Hamburg, C. H. Beck, München 1983, DM 19,80

Mit dem "Jahrbuch Dritte Welt" hat das Deutsche Úbersee-Institut eine bemerkenswerte Reihe eröffnet, die künftig jährlich fortgesetzt werden soll. Mit seiner Vielzahl an unterschiedlichen Einzelbeiträgen wird es seinem Anspruch einer schwerpunktmäßigen, regionenübergreifenden Information über im Jahresverlauf 1982 für die Länder der Dritten Welt besonders bedeutsame, politische, wirtschaftliche und gesellschaftliche Ereignisse und Probleme weitgehend gerecht. Eingeleitet wird das in fünf Teile gegliederte Jahrbuch durch einen Beitrag der Redakteure Joachim Betz und Volker Matthies "Die Dritte Welt im Berichtsjahr" (S. $11 \mathrm{ff}$.) in Teil I - Editorial. Sie stellen die Zusammenhänge der einzelnen im Jahrbuch enthaltenen Beiträge dar. In dem allgemein politischen Uberlegungen vorbehaltenen Jahrbuchforum - Teil II - hält Erhard Eppler in seinem Beitrag "Frieden - Okologie - Entwicklung" (S. 20 ff.) plastisch die Folgen einer technokratisch orientierten Entwicklungspolitik - wie sie zu seiner Amtszeit als Bundesminister für wirtschaftliche Zusammenarbeit eingeleitet wurde - verbunden mit einem die Stabilität der Dritten Welt gefährdenden Rüstungsexport vor Augen. Dem stellt er auf dem Herder'schen Entwicklungsbegriff "Entfaltung aus eigenem Ansatz und eigener Kraft" (S. 27) aufbauend eine Friedensvision humaner Konfliktlösung als Ziel jeder politischen Kultur entgegen. In diesem Zusammenhang ist der Beitrag Michael Brzoska's "Neue Richtlinien für den Waffenexport aus der Bundesrepublik Deutschland in die Dritte Welt" (S. 87 ff.) in dem eigens der Bundesrepublik Deutschland vorbehaltenen Teil IV von besonderem Interesse. Entgegen der sonst häufig üblichen quantitativen Angaben zum Rüstungsexport legt Brzoska die rechtlichen Grundlagen des bundesdeutschen Waffenexports und deren Schwachstellen vor allem bei der Kontrolle des Exports von Rüstungsgütern aus Kooperationsprojekten und der Lizenzvergabe dar. Begünstigt wurde dieser Zustand durch die faktische Außer-Kraft-Setzung der den Waffenexport regelnden Richtlinien von 1971 seit 1975. "Eine 'Wender ist - nach den Worten Brzoska's - mit den neuen Richtlinien nicht vollführt worden - eher wurde die bisherige Praxis mit einigen Abweichungen und viel Spielraum in Worte gefaßt" (S. 99). Dieser Spielraum macht sich vor allem in dem ohne Vetorecht ausgestatteten Konsultationsverfahren beim Export von in Kooperation hergestellten Rüstungsgütern wie auch bei der sehr weit gehaltenen sogenannten "Chile-Klausel", nach der die "innere Lage des betreffenden Landes" der Waffenlieferung nicht entgegenstehen darf, bemerkbar; während die in anderen westlichen Ländern übliche Anhörung des Entwicklungshilfeministeriums bei der Genehmigung von Waffenlieferungen auch nach den Richtlinien von 1982 nicht vorgesehen ist.

Aus dem "überregionalen Beiträgen" gewidmeten Teil III des Jahrbuches sind die Beiträge von Joachim Betz "Verschuldenskrise der Dritten Welt" (S. 30 ff. $)^{1}$ und Al-

1 Vgl. auch J. Betz, VRU 15 (1982), S. $455 \mathrm{f}$. 
phons Studier "Die neue Weltmeeresordnung" (S. $57 \mathrm{ff}$.) besonders hervorzuheben. Betz stellt die selten so augenscheinlich ins rechte Licht gerückte Dimension der Verschuldenskrise nur einiger weniger, erstaunlicherweise vorwiegend Schwellen- und rohstoffreicher Entwicklungsländer dar, gegenüber der die Verschuldung und der Schuldendienst armer Länder vergleichsweise gering ist und unproblematisch verläuft. Studier widmet sich ausführlich der rechtlichen Ausgestaltung der neuen Weltmeeresordnung, die entgegen ihrer ursprünglichen Intention, "den Meeresbereich jenseits der . . geltenden Hoheitsgrenzen zum gemeinsamen Menschheitserbe - Common Heritage of Mankind - zu erklären" (S. 59), durch die weite Ausdehnung von Wirtschaftszone und Festlandsockel eine nationale Aneignung von mehr als $40 \%$ der Ozeane durch nur zehn Staaten, unter ihnen die Industrienationen: USA, UdSSR, Frankreich, Großbritannien, ermöglicht, während die anfänglich beabsichtigte Stärkung der Meeresbodenbehörde bereits im Hinblick auf ihre finanzielle Ausstattung (anfänglich sollten ihr ca. 6 Mrd. US-\$ zur Verfügung stehen, tatsächlich sind es nun aber lediglich 150-250 Mill. US-\$) auf der Strecke blieb. Vergleichsweise blaß sind demgegenüber die Beiträge von Hanns W. Maull "Von der Verknappungskrise zur Olschwemme" (S. 45 ff.) und Franz Nuscheler "Reaganism und Dritte Welt: Neue Rethorik oder entwicklungspolitische Wende?" (S. 73 ff.), die einem einigermaßen regelmäßigen Zeitungsleser weitgehend Bekanntes wiedergeben.

Hervorzuheben ist der den größten Teil des Jahrbuches umfassende Teil V mit ausschließlich "regionalen Beiträgen". Hier gibt Theodor Hauf, "Der Krieg im Libanon" (S. 101 ff.), einen aufschlußreichen Einblick in die von dem "Klischee vom Konflikt zwischen rechten Christen und linken Muslimen" (S. 104) behaftete innenpolitische Lage des Landes, die wesentlich von der jüngst wieder ins Bewußtsein gerufenen Auseinandersetzung zwischen Syrern und Palästinensern geprägt ist. Rüdiger Robert stellt in seinem Beitrag "Der iranisch-irakische Krieg" (S. 119 ff.) Allianzen und Zusammenhänge innerhalb der arabischen Staaten und die Verstrickung der beiden Weltmächte in diesen Konflikt, der aus den bundesdeutschen Medien weitgehend verschwunden ist, heraus. Einem ebenfalls in der Berichterstattung vernachlässigten Bereich widmet sich Peter Schier "(K)eine Lösung für Kambodscha?" (S. 185 ff.). Kritisch wird hier das vietnamesische Bestreben nach einer "schrittweisen, politischen und wirtschaftlichen Verschmelzung" mit Laos und Kambodscha gesehen und die demgegenüber einerseits sehr skeptische Haltung der Volksrepublik China, andererseits wohlwollende Haltung der Sowjetunion untersucht. Von überwiegend historischem Interesse ist demgegenüber der Beitrag Michael Ehrkes "Argentinien und der Krieg im Südatlantik" (S. 134 ff.). Auch Mechthild Minkner "Krisenherd Zentralamerika" (S. 150 ff.) bringt wahrscheinlich wegen des bestehenden, besonders breiten Interesses - nicht wesentlich Neues. Einen interessanten Einblick in die wirtschaftliche und politische Situation Mexikos und Tanzanias geben Rainer Godau "Wirtschaftlicher Kollaps in Mexiko" (S. 170 ff.) und Rolf Hofmeier "Tanzania - 'Entwicklungsmodell oder Entwicklungsbankrott?“ (S. 204 ff.), wobei vor allem Hofmeier wegen der vergleichsweise starken 
inneren Stabilität des Landes einen Erfolg der Entwicklungsstrategie des Ujamaa-Sozialismus auch angesichts der augenblicklichen wirtschaftlichen Schwierigkeiten Tanzanias noch nicht für ausgeschlossen ansieht. Aus aktuellem Anlaß - dem Streit um die Aufnahme der "Demokratisch-Arabischen Republik Sahara" (DARS) - widmet sich Goswin Baumhögger in seinem Beitrag "Die Organisation der Afrikanischen Einheit (OAU) am Scheidewege/" (S. $221 \mathrm{ff}$.) u. a. einer möglichen Zukunftsperspektive der $\mathrm{OAU}$, die nach ihrem in den letzten Jahren verstärkten Engagement für die wirtschaftliche Entwicklung und die Menschenrechte im Bereich einer "schlagkräftigen Dienstleistungsorganisation" (S. 235) liegt.

$\mathrm{Zu}$ loben ist das Jahrbuch wegen seiner durchweg klaren und verständlichen Sprache, durch die eine differenzierte Betrachtung der Beiträge nicht beeinträchtigt wird und die ihm einen weiten Leserkreis eröffnet, sowie wegen seinem schnellen Erscheinen auf aktuellem Stand! Gut ausgewählt sind die Beiträge - selbst wenn einem die Beiträge Maulls und Nuschelers etwas überflüssig erscheinen mögen, so können sie doch für andere ausgesprochen aufschlußreich sein, bedenkt man den breiten Adressatenkreis des Jahrbuches "über die Wissenschaftler, Politiker und Institutionen hinaus vor allem an die breite, entwicklungspolitisch interessierte Offentlichkeit“ (S. 5; Ziff. 7). Einer vertiefenden Beschäftigung hilfreich sind die den Beiträgen angefügten Literaturhinweise. Indes sind sie zu gering, eine wissenschaftliche Auseinandersetzung zu befruchten. Doch sollte das Jahrbuch als aktuelles Nachschlagewerk in Universitäts- und Institutsbibliotheken nicht fehlen, da es einen guten Einstieg in die verschiedenen Problembereiche bietet. Aus diesem Grunde ist es besonders für Einrichtungen der Weiterbildung von unschätzbarem Wert. Auf das Jahrbuch 2 (1984) darf man gespannt sein!

Ulrich Werwigk

\section{Justin Stagl}

Kulturanthropologie und Gesellschaft - Eine wissenschaftssoziologische Darstellung der Kulturanthropologie und Ethnologie

2., durchgesehene, verbesserte und um ein Nachwort vermehrte Auflage, Dietrich Reimer Verlag, Berlin, 1981, 195 S.

Wenn ein Buch sechs Jahre nach seinem ersten Erscheinen neu aufgelegt wird, hat es offensichtlich in der Leserschaft Beachtung gefunden. Was also ist das für ein Werk, das "Kulturanthropologie und Gesellschaft" heißt und in manchen Seminarbibliotheken als "verschwunden" abgeschrieben werden muß?

Der Autor ist Professor der Soziologie an der Universität Bonn; seine Veröffentlichungen liegen jedoch schwerpunktmäßig bei der Ethnologie. Wie es der Untertitel zur zweiten Auflage bereits ausdrückt, handelt es sich bei der vorliegenden Arbeit um eine 